\title{
手性磷酰胺配体不对称催化二芳基甲醇类化合物的合成
}

\author{
郭庆君* \\ (济宁学院化学与化工系 山东曲阜 273155)
}

\begin{abstract}
摘要 为了获得具有重要生理活性的手性二芳基甲醇类化合物, 以 $R, R-1,2$-环己二胺为原料获得了一系列磷酰胺配体, 并系统考察了该类配体在芳基烷基锌对芳香酫的不对称加成反应中的催化活性，在优化的反应条件下，即在 $30 \mathrm{~mol} \%$ 磷酰胺配体 $N-((1 R, 2 R)-2$-(异丙基氨基)环己基)- $P, P$-二苯基磷酰胺(9c)存在下, 可以高达 $94 \%$ 的 $e e$ 值(对映体过量值)及 大于 $90 \%$ 收率获得相应的手性二芳基甲醇类化合物. 尽管催化剂用量较大, 在该体系中, 配体可非常方便回收再利用. 同时, 对反应机理进行了推测, 认为反应过程所形成的四元过渡态和六元过渡态, 有利于提高反应的对映选择性. 关键词 手性磷酰胺类配体; 手性二芳基甲醇类化合物; 不对称催化
\end{abstract}

\section{Asymmetric Synthesis of Diarylmethanols by Chiral Phosphoramide Ligands Catalysts}

\author{
Guo, Qingjun* \\ (Key Laboratory of Inorganic Chemistry in Universities of Shandong, Department of Chemistry and \\ Chemical Engineering, Jining University, Qufu 273155, Shandong)
}

\begin{abstract}
In order to improve the application of chiral phosphoramide ligands in catalytic asymmetric reactions, thiophosphoramide, which was synthesized from trans-1,2-cyclohexanediamine was used as a catalyst to synthesize chiral diarylmethanol compounds through addition reaction. The catalytic activity of the ligand in the asymmetric addition reaction of the arylalkyl zincs to the aromatic aldehyde can be as high as $94 \%$ ee under the optimized reaction conditions in the presence of 30 mol\% phosphoramide ligand $N$-((1R,2R)-2-(isopropylamino)cyclohexyl)- $P, P$-diphenylphosphinic amide (9c) and the corresponding chiral diarylmethanol compound was obtained with the yields of $>90 \%$. Despite the large amount of catalyst, the ligand is very convenient to recycle and reuse in this system. At the same time, the reaction mechanism was speculated, and it is believed that the quaternary transition state and the six-element transition state formed by the reaction process are beneficial to improve the enantioselectivity of the reaction.
\end{abstract}

Keywords chiral thiophosphoramide ligand; diarylmethanols; asymmetric catalysis

手性是自然界最重要的属性之一 ${ }^{[1]}$. 绝大多数的药 物是由手性化合物组成的, 拥有药理作用的药物一般都 是手性专一的, 如具有抗白血病作用的 cochleamycin A 及具有抗菌作用的(一)-Tetrecycline(图 1). 相反手性的 化合物可能不会具备药理活性或者有严重的副作用. 具 有二芳基甲醇分子构架的化合物是很多具有生物活性 的天然产物和药物的重要组成构架, 例如 cizolirtine $(\mathbf{1})^{[2]} 、$ neobenodine $(2)^{[3 \mathrm{a} \sim 36]} 、$ carbinoxamine $(\mathbf{3})^{[4]}$ 和 orphenadrine (4) $)^{[2,5]}$ (图 1). 另外, 一些药物是手性二芳基甲 醇衍生物, 例如化合物 $\mathbf{5}^{[6]}$ 和 $\mathbf{6}^{[7]}$ (图 1). 获得该类化合物 的常用方法是通过亲核取代反应来合成, 该方法所获得 的二芳基甲醇往往无法保证得到较好的光化学活性. 近
年来, 通过添加手性配体来催化芳香基化合物对醛进行 不对称加成, 可以较为有效地合成手性专一的醇类分子 构架.

手性配体催化芳基金属试剂与芳香醛或脂肪醛发 生不对称加成反应来合成手性二芳基化合物越来越受 到人们的重视 ${ }^{[8]}$. 目前已有报道的芳基金属试剂包括芳 基铝试剂 $[9]$ 、芳基格式试剂 ${ }^{[10]}$ 、芳基硅烷 ${ }^{[11]}$ 、芳基钛试 剂 ${ }^{[12]}$ 以及芳基锂试剂 ${ }^{[13]}$ 等. 得益于芳基锌试剂的低反 应活性，其在催化合成手性二芳基化合物的过程中，反 应条件温和, 底物范围较广. 因此催化芳基锌试剂与芳 香醛发生不对称加成反应来合成手性二芳基化合物越 来越受到人们的重视. 早在 2002 年, Bolm 等 ${ }^{[14]}$ 以硼酸

* Corresponding author. E-mail: guoqingjun2005@126.com

Received February 24, 2019; revised March 29, 2019; published online May 21, 2019. 
为芳

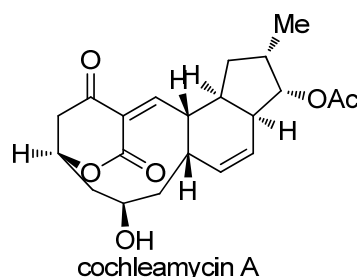

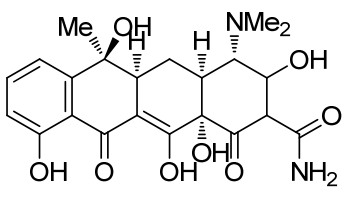

(-)-Tetracycline<smiles>CN(C)CCOC(c1ccccc1)c1ccnn1C</smiles><smiles>Cc1ccc(C(OCCN)c2ccccc2)cc1</smiles>

2<smiles>Cc1ccc(C(OCCN)c2ccccn2)cc1</smiles>

3<smiles>Cc1ccccc1C(OCCN(C)C)c1ccccc1</smiles><smiles>COc1ccc(C(Cc2ccncc2)c2ccccc2)cc1CC1CCCC1</smiles><smiles>CN(C)CCc1ccc(Cl)cc1</smiles>

6

图 1 手性药物及部分含有二芳基甲醇构架

Figure 1 Structure of biologically active diarylmathanol derivative

基原，用手性二茂铁配体催化合成手性二芳基甲醇类化 合物. 嗍酸试剂廉价易得, 且绝大多数硼酸试剂均有市 售, 因此由嗍酸作为芳基原催化合成手性二芳基化合物 越来越受到人们的青睐 ${ }^{[15]}$. 目前面临的挑战是寻找更 为简单且高效的配体.

手性磷酰胺配体在催化领域的应用冊庸置疑, 该类 配体不仅在催化不对称反应中有很好的适用性 ${ }^{[16]}$, 在 手性酸的对映体识别中也有着很好的适用性 ${ }^{[17]}$. 为了 拓宽手性磷酰胺- $\mathrm{Zn}(\mathrm{II})$ 在不对称催化领域的应用, 下面 介绍一种以环已基磷酰胺作为配体, 芳基锌试剂与芳香 醛发生不对称加成反应生成高产率、高 $e e$ 值的二芳基 甲醇类化合物的反应. 手性磷酰胺的配体 $9 \mathrm{c}$ 的合成见 Scheme 1.

\section{1 结果与讨论}

\section{1 手性配体的篮选}

对甲基苯甲醛和苯硼酸为原料进行了配体的篎选 (表 1). 首先使用苯硼酸及甲基锌试剂在甲苯中加热至 $60{ }^{\circ} \mathrm{C}$ 摚拌 $12 \mathrm{~h}$ 得到苯基甲基锌试剂, 之后在相应配体 的催化条件下进行催化反应. 由表 1 Entry 1 可以看到, 使用以奎宁合成的手性配体 7 进行催化反应, 虽然产率 可达到 $83 \%$, 但 $e e$ 值仅为 $46 \%$. 以反式-1, 2-二苯基乙二 胺为原料合成的手性配体 $\mathbf{8}$ 催化该反应, 产率和 $e e$ 值均 有一定的提高, 但 $e e$ 值仅为 $68 \%$ (表 1, Entry 2). 以反 式-1,2-环已二胺为原料合成的手性配体 9a 催化该反应
时，可将反应的产率提高到 96\%,ee 值仅为 29\%(表 1, Entry 3). 在此基础上对该配体进行相应的修饰, 将氨基

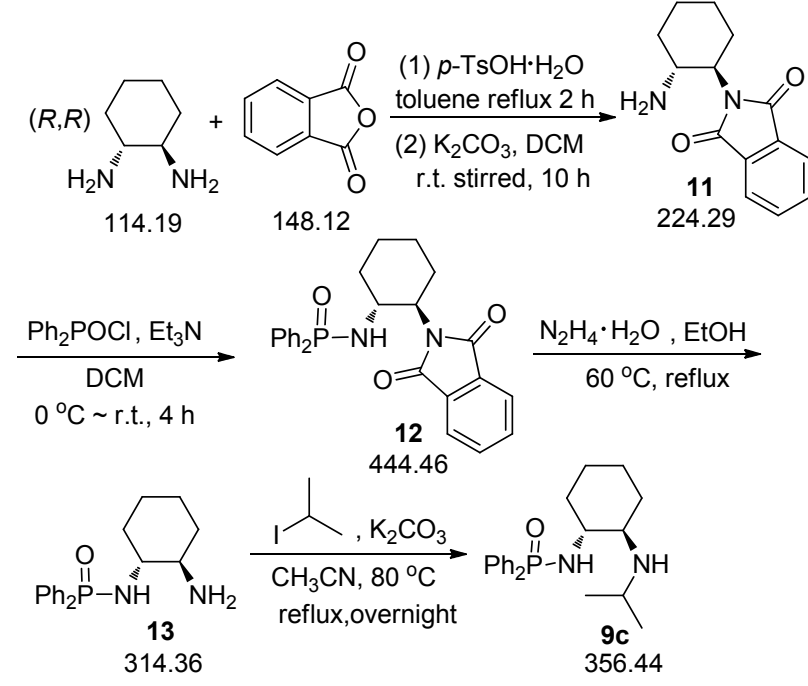

图式 1 配体 $9 \mathrm{c}$ 的合成路线

Scheme 1 Synthesis route of ligand $9 \mathrm{c}$

表 1 手性配体的篮选 ${ }^{a}$

Table 1 Screening of chiral ligand

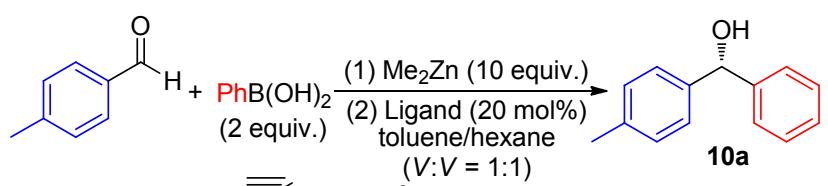

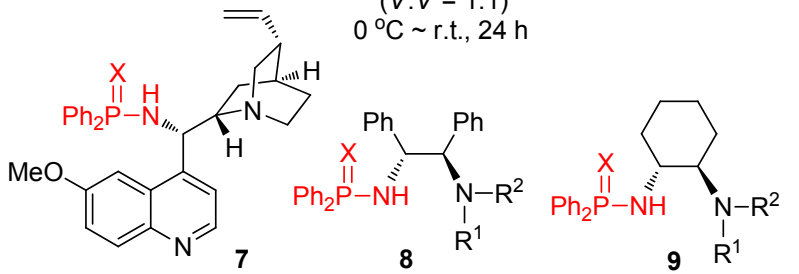

\begin{tabular}{cccccc}
\hline Entry & Ligand & $\mathrm{X}$ & $\mathrm{NR}^{1} \mathrm{R}^{2}$ & Yield $^{b} / \%$ & $e e^{c} / \%$ \\
\hline 1 & $\mathbf{7}$ & $\mathrm{O}$ & - & 83 & 46 \\
2 & $\mathbf{8}$ & $\mathrm{O}$ & $\mathrm{NH}^{i} \mathrm{Pr}$ & 89 & 68 \\
3 & $\mathbf{9 a}$ & $\mathrm{O}$ & $\mathrm{NHBn}$ & 96 & 29 \\
4 & $\mathbf{9 b}$ & $\mathrm{O}$ & $\mathrm{NEt}_{2}$ & 84 & 56 \\
5 & $\mathbf{9 c}$ & $\mathrm{O}$ & $\mathrm{NH}^{i} \mathrm{Pr}$ & 93 & 78 \\
6 & $\mathbf{9 d}$ & $\mathrm{S}$ & $\mathrm{NH}^{i} \mathrm{Pr}$ & 88 & 76 \\
\hline
\end{tabular}

${ }^{a}$ Unless otherwise noted, all reactions were carried out with benzaldehyde on 0.5 mmol scale. ${ }^{b}$ Isolated yields. ${ }^{c}$ Determined by chiral GC or HPLC analysis and the absolute configuration was assigned by comparison of the sign of specific rotation value with the literature value.

上的取代基进行更换. 由表 1 中 Entries 3 6 可以看到, 当氨基上为单异丙基取代时，产物的产率保持在 93\%, $e e$ 值提高到 $78 \%$. 由此得出以下结论：最佳配体为手性 磷酰胺配体 $9 \mathbf{c}$.

\section{2 最佳反应条件的选择}

为了验证最佳反应条件，分别对反应溶剂的类型、 反应温度、锌试剂的种类以及配体的用量进行了系统的 
研究(表 2). 通过表 2 的数据可以发现, 最佳反应溶剂为 体积比为 $1: 1$ 的甲苯与正己烷混合溶剂(表 2, Entries $1 \sim 4)$, 当使用甲苯与正己烷的混合溶剂时, 所得产物 的产量可达到 $93 \%$, ee 值为 78\%. 在此基础上, 又对反 应温度进行了相应的研究, 当温度从 $0 \sim 25{ }^{\circ} \mathrm{C}$ 降至 $-40{ }^{\circ} \mathrm{C}$ 时, 产率虽然降低到 $89 \%$, 但产物的 ee 值却升 高到 $86 \%$ (表 2, Entries 5 8). 之后又对锌试剂的类型进 行了研究, 将甲基锌试剂更换为乙基锌试剂后, 产率和 $e e$ 值均有少许的提高(表 2, Entries 8,9). 最后对配体的 用量进行了研究, 研究结果表明, 当配为 $30 \mathrm{~mol} \%$ 时, 可保证产率为 $93 \%$ 的同时, ee 值提高到 $94 \%$ (表 2, Entries $9 \sim 11)$. 虽然当配体为 $40 \mathrm{~mol} \%$ 时, 产率和 $e e$ 值与 30 $\mathrm{mo} \%$ 时类似, 但考虑到配体使用的经济性, 最终选择配 体的用量为 $30 \mathrm{~mol} \%$. 由此得到最佳反应条件是：以甲 苯与正己烷的混合溶剂(体积比为 $1: 1$ ) 作为反应溶剂, 反应温度为 $-40{ }^{\circ} \mathrm{C}$, 锌试剂为乙基锌试剂, 配体使用 量为 $30 \mathrm{~mol} \%$.

表 2 反应条件的优化

Table 2 Optimization of the reaction condition

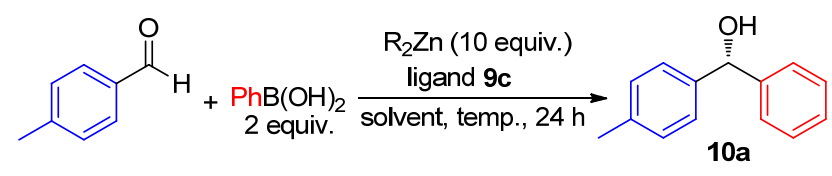

\begin{tabular}{cccclcc}
\hline Entry & $\begin{array}{c}9 c / \\
\text { mol\% }\end{array}$ & $\mathrm{R}$ & $\begin{array}{c}\text { Temp./ } \\
{ }^{\circ} \mathrm{C}\end{array}$ & \multicolumn{1}{c}{ Solvent } & Yield $/ \%$ & $e e^{c} / \%$ \\
\hline 1 & 20 & $\mathrm{Me}$ & $0 \sim 25$ & Toluene & 89 & 74 \\
2 & 20 & $\mathrm{Me}$ & $0 \sim 25$ & Hexane & 85 & 70 \\
$3^{d}$ & 20 & $\mathrm{Me}$ & $0 \sim 25$ & Hexane/MTBE & 53 & 71 \\
$4^{d}$ & 20 & $\mathrm{Me}$ & $0 \sim 25$ & Hexane/THF & Trace & N.D. ${ }^{e}$ \\
$5^{d}$ & 20 & $\mathrm{Me}$ & $0 \sim 25$ & Toluene/hexane & 93 & 78 \\
$6^{d}$ & 20 & $\mathrm{Me}$ & -10 & Toluene/hexane & 90 & 80 \\
$7^{d}$ & 20 & $\mathrm{Me}$ & -20 & Toluene/hexane & 90 & 82 \\
$8^{d}$ & 20 & $\mathrm{Me}$ & -40 & Toluene/hexane & 89 & 86 \\
$9^{d}$ & 20 & $\mathrm{Et}$ & -40 & Toluene/hexane & 90 & 89 \\
$10^{d, f}$ & 30 & $\mathrm{Et}$ & -40 & Toluene/hexane & 93 & 94 \\
$11^{d}$ & 40 & $\mathrm{Et}$ & -40 & Toluene/hexane & 93 & 92 \\
\hline
\end{tabular}

${ }^{a}$ Unless otherwise noted, all reactions were carried out with benzaldehyde on 0.5 mmol scale. ${ }^{b}$ Isolated yields. ${ }^{c}$ Determined by chiral GC or HPLC analysis and the absolute configuration was assigned by comparison of the sign of specific rotations with the literature values. ${ }^{d}$ The ratio of the solvent volume was $1: 1 .{ }^{e}$ Not determined. ${ }^{f}$ The chiral ligand can be recovered with $98 \%$ yield

\section{3 反应底物的拓展}

在得到最佳配体与反应条件后, 研究了该不对称反 应的普适性，见表 3. 由表 3 的 Entries 1 10 可以看到， 无论芳香醛有吸电子基还是给电子基, 使用该催化条件 进行催化反应均能得到较高的产率及 $e e$ 值, 产率可从 $81 \%$ 到 $98 \%$, ee 值可从 $82 \%$ 到 $94 \%$. 将芳香醛更换成 1蒜醛及 2-蒜醛时, 该催化体系也能使所得产物的产率和 $e e$ 值高达 $80 \%$ 以上(表 3, Entries 11, 12)；当固定芳香醛 为苯甲醛，对硼酸上的芳基进行更改和修饰，实验结果 表明，该催化体系下产物产率达 $90 \%$, ee 值达 $88 \%$ 以上 (表 3, Entries 13 15); 分别对芳香醛和芳基硼酸上的芳 基同时进行修饰，所得产物的产率及 $e e$ 值也很好(表 3, Entries 16 21); 最后将芳香醛更换为 2-噻吩甲醛时, 产物的产率虽然达 90\%, 但 $e e$ 值仅为 $53 \%$, 因此该催化 体系并不适合于 2-噻吩甲醛. 通过实验可以发现, 当体 系中有酯基存在时也不会对该催化体系产生影响，酯基 在催化过程中不会被破坏.

\section{4 反应机理推测}

反应机理如 Scheme 2 所示，乙基锌试剂首先与硼 酸反应生成芳基烷基锌. 当加入手性磷酰胺配体时，环 己基二胺配体首先与锌试剂反应生成相应的配体-Zn(II) 配合物, 因为 $\mathrm{Zn}(\mathrm{II})$ 的存在导致磷酰胺配体上磷氧双键 的路易斯酸性增强，促进了芳基烷基锌与芳香醛加成反 应的进行. 在进行加成反应时, 芳香醛上的碳氧双键与 芳基烷基锌上的芳基-Zn(II)发生共轭，形成四元过度环， 与此同时醛基上的氧、芳基烷基锌中的锌原子，磷酰胺 上的磷原子、氧原子、氮原子与锌原子形成六元过渡态， 促使加成反应的进行. 因为四元过渡态和六元过渡态的 存在，导致芳基烷基锌中的芳基与芳香醛中的芳基之间 存在一定的空间位阻作用. 也正是因为该作用的存在, 才使得所得二级醇拥有良好的绝对构型.

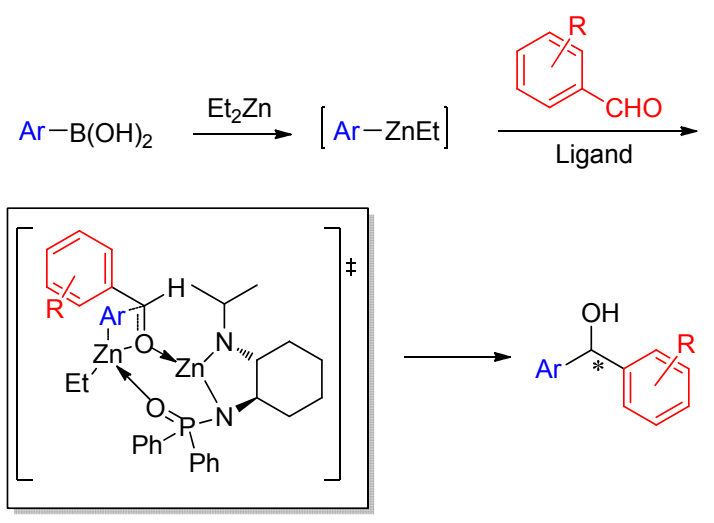

图式 2 反应机理推测

Scheme 2 Speculation of reaction mechanism

\section{2 结论}

通过以较为廉价的手性环己基二胺为起始原料，经 过几步简单的反应, 合成了手性磷酰胺配体 9c. 在一 $40{ }^{\circ} \mathrm{C}$ 下 9c 催化芳基烷基锌试剂与芳香醛进行加成反 应，合成了具有重要药物分子骨架的二芳基甲醇类化合 物. 该方法不仅可以使产率保持在一个较高的值, 也可 使 $e e$ 较为理想, 对反应机理进行了解释. 除此之外, 该 
表 3 底物的拓展 ${ }^{a}$

Table 3 Asymmetric arylation of aromatic aldehydes

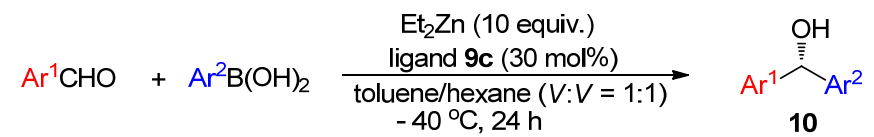

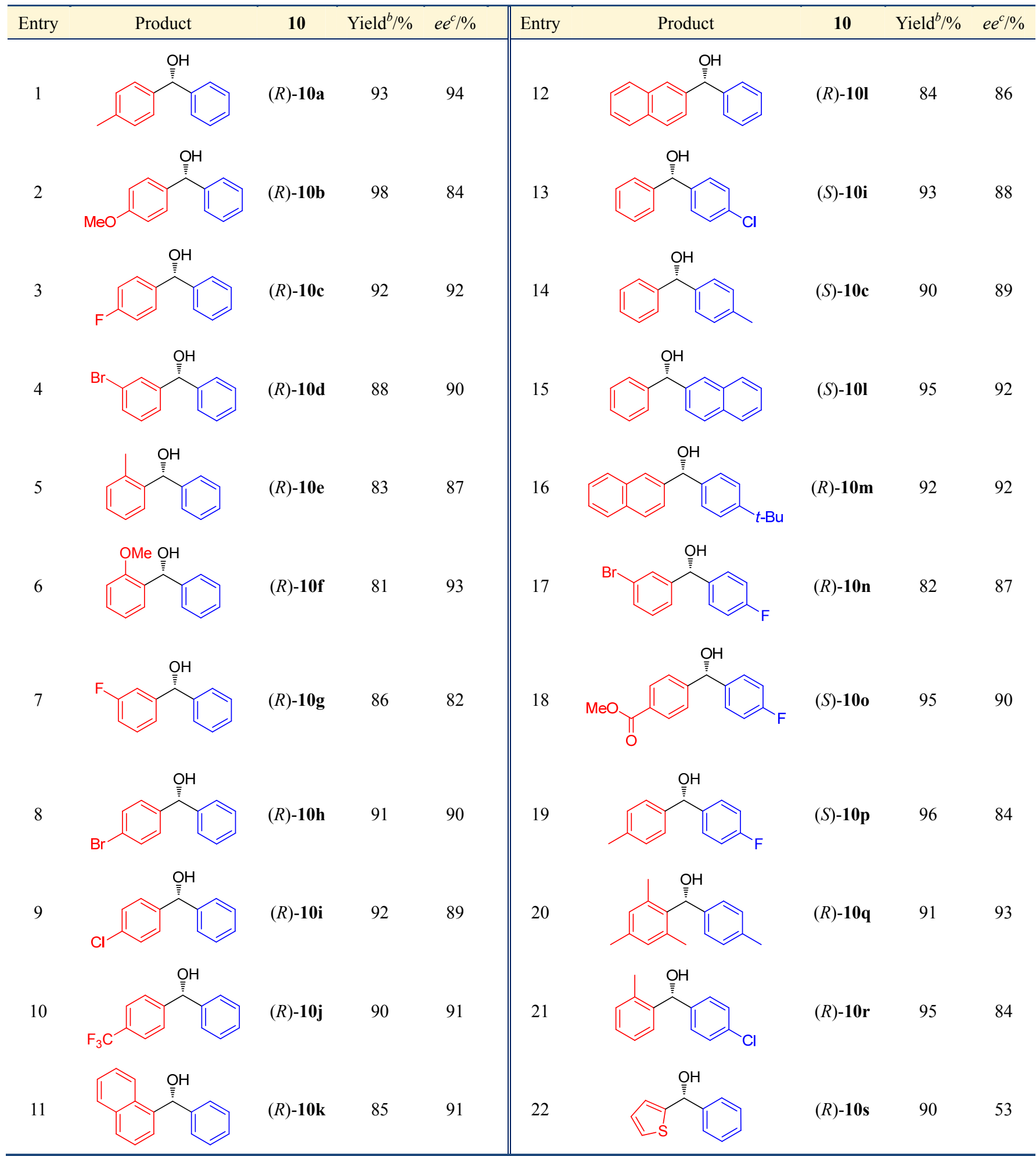

${ }^{a}$ 除非特殊说明, 所有的反应均以相应的醛为 $0.5 \mathrm{mmol}$ 的量进行; ${ }^{b}$ 分离收率; ${ }^{c}$ 数值经过 $\mathrm{GC}$ 或者 $\mathrm{HPLC}$ 确定, 绝对构型经过比旋光度与文献报道值对比确 定.

方法对体系中的酯基没有影响，且所使用的配体几乎可 以定量回收，并可循环使用. 


\section{3 实验部分}

\section{1 仪器与试剂}

${ }^{1} \mathrm{H}$ NMR (400 MHz), ${ }^{13} \mathrm{C}$ NMR (100 MHz) 和 ${ }^{31} \mathrm{P}$ NMR $(162 \mathrm{MHz})$ 谱图均以 Bruker DR $\times 400$ 型核磁共振 仪测试所得, 所用氞代试剂均为 $\mathrm{CDCl}_{3}$, 化学位移的基 准为 $\mathrm{CDCl}_{3}$, 氢谱化学位移是 $\delta 7.26$, 碳谱化学位移是 $\delta$ 77.0. 若无特殊说明, 所用的试剂均是商业购买获得, 溶剂均按照文献报道的相关方法进行干燥. 旋光值均用 旋光仪测得. 气相数据分析均以带有 FID 检测器以及手 性硅胶毛细管柱(Chirasil Dex CB column, $25 \mathrm{~m} \times 0.32$ $\mathrm{mm} \times 0.25 \mathrm{~mm}$ film thickness)的气相色谱仪测试得到, 高效液相色谱(HPLC)数据以岛津高效液相色谱仪及手 性 OD-H 柱, AD-H 柱, OJ-H 柱和 AS-H 柱测试得到. 手 性配体 $7^{[18]}, 8^{[19]}$, 以及 $9 a, 9 b$ 和 $9 d^{[17]}$ 的合成参考文献方 法获得.

\section{2 手性配体 $9 c$ 的合成}

3.2.1 单邻苯二甲酸酐保护的 $(R, R)$-环已基二胺 $(\mathbf{1 1})$ 的合成

按照文献[20]报道的方法合成. m.p. $249 \sim 252{ }^{\circ} \mathrm{C}$ (lit. ${ }^{[20]}$ : m.p. $\left.249 \sim 253{ }^{\circ} \mathrm{C}\right) ;{ }^{1} \mathrm{H}$ NMR $\left(400 \mathrm{MHz}, \mathrm{CDCl}_{3}\right)$ $\delta: 7.88 \sim 7.80(\mathrm{~m}, 2 \mathrm{H}), 7.75 \sim 7.67(\mathrm{~m}, 2 \mathrm{H}), 3.84 \sim 3.78$ $(\mathrm{m}, 1 \mathrm{H}), 3.46 \sim 3.39(\mathrm{~m}, 1 \mathrm{H}), 2.26 \sim 2.15(\mathrm{~m}, 1 \mathrm{H}), 2.08 \sim$ $2.03(\mathrm{~m}, 1 \mathrm{H}), 1.86 \sim 1.76(\mathrm{~m}, 3 \mathrm{H}), 1.51 \sim 1.16(\mathrm{~m}, 5 \mathrm{H})$; ${ }^{13} \mathrm{C}$ NMR $\left(100 \mathrm{MHz}, \mathrm{CDCl}_{3}\right) \delta: 168.76,133.83,131.91$, 123.12, 58.57, 50.84, 36.72, 29.32, 25.64, 25.10; IR (KBr) $v: 3465,3055,2934,2866,1711,1647,1554,1369,846$, 831, 715; HMRS calcd for $\mathrm{C}_{14} \mathrm{H}_{16} \mathrm{~N}_{2} \mathrm{O}_{2}$ 244.1212, found 244.1209

\section{2 .2 化合物 12 的合成}

在 $100 \mathrm{~mL}$ 反应瓶中加入上步所得化合物 11 (14.66 $\mathrm{g}, 60 \mathrm{mmol})$ 和溶剂二氯甲烷 $(200 \mathrm{~mL})$, 搅拌全溶, 再将 三乙胺(15.16 g, $150 \mathrm{mmol}$ )加入到反应体系中. 室温下 搅拌 $10 \mathrm{~min}$, 搅拌完毕将反应瓶放于冰水浴中. 将用 $100 \mathrm{~mL}$ 二氯甲烷稀释后的二苯基膦酰氯(21.28 g, 90 $\mathrm{mmol}$ )溶液逐滴加入反应瓶中, 加入完毕, 将反应瓶从 冰浴中取出并升至室温. 在室温条件下继续摚拌反应 4 h. 反应完毕, 将反应瓶放入冰水浴中, 逐滴滴加 $40 \mathrm{~mL}$ 水对体系进行淬灭, 淬灭结束后使用 $40 \mathrm{~mL}$ 二氯甲烷萃 取三次, 合并有机相并用无水硫酸钠进行干燥. 将干燥 完成的有机相进行减压浓缩, 最后将所得粗品进行柱层 析法 $[V$ (乙酸乙酯 $) / V($ 正己烷 $)=1 / 1$ ]分离提纯得单邻苯二 甲酸䣶保护的 $(R, R)$-环已基二胺衍生物 $12,22.40 \mathrm{~g}$, 产 率 84\%. m.p. $193 \sim 194{ }^{\circ} \mathrm{C}$; $[\alpha]_{\mathrm{D}}^{22.0}-41.5$ (c 2.00, $\left.\mathrm{CH}_{2} \mathrm{Cl}_{2}\right)\left(\right.$ lit. ${ }^{[21]}$ : m.p. $193 \sim 194{ }^{\circ} \mathrm{C},[\alpha]_{\mathrm{D}}^{22.0}-41.2$ (c 2.00,
$\left.\left.\mathrm{CH}_{2} \mathrm{Cl}_{2}\right)\right) ;{ }^{1} \mathrm{H}$ NMR $\left(400 \mathrm{MHz}, \mathrm{CDCl}_{3}\right) \delta: 1.20 \sim 1.40(\mathrm{~m}$, $3 \mathrm{H}), 1.65 \sim 1.85(\mathrm{~m}, 3 \mathrm{H}), 2.26 \sim 2.42(\mathrm{~m}, 2 \mathrm{H}), 2.90 \sim 3.00$ $(\mathrm{m}, 1 \mathrm{H}), 3.50 \sim 3.68(\mathrm{~m}, 1 \mathrm{H}), 3.85 \sim 4.00(\mathrm{~m}, 1 \mathrm{H}), 7.00 \sim$ $7.13(\mathrm{~m}, 2 \mathrm{H}), 7.23 \sim 7.31(\mathrm{~m}, 1 \mathrm{H}), 7.32 \sim 7.39(\mathrm{~m}, 2 \mathrm{H})$, $7.40 \sim 7.53(\mathrm{~m}, 3 \mathrm{H}), 7.65 \sim 7.75(\mathrm{~m}, 4 \mathrm{H}), 7.75 \sim 7.85(\mathrm{~m}$, $2 \mathrm{H}) ;{ }^{13} \mathrm{C}$ NMR $\left(100 \mathrm{MHz}, \mathrm{CDCl}_{3}\right) \delta: 24.8,25.2,28.7,37.0$ (d, $J=2.5 \mathrm{~Hz}), 51.6,56.2(\mathrm{~d}, J=5.9 \mathrm{~Hz}), 122.9,128.0$ (d, $J=12.8 \mathrm{~Hz}), 128.2(\mathrm{~d}, J=12.6 \mathrm{~Hz}), 131.4(\mathrm{~d}, J=38.4 \mathrm{~Hz})$, 131.5 (d, $J=9.5 \mathrm{~Hz}), 131.7$ (d, $J=7.6 \mathrm{~Hz}), 131.8$ (d, $J=$ $9.1 \mathrm{~Hz}), 133.0(\mathrm{~d}, J=5.8 \mathrm{~Hz}), 133.6,168.3,168.6 ;{ }^{31} \mathrm{P}$ NMR (162 MHz, $\left.\mathrm{CDCl}_{3}\right) \delta: 22.0$; IR (KBr) v: 3051, 2928, $2855,1715,1619,1558,1279,754,717$; HMRS calcd for $\mathrm{C}_{26} \mathrm{H}_{25} \mathrm{~N}_{2} \mathrm{O}_{3} \mathrm{P}$ 444.1603, found 444.1605. Anal. calcd for $\mathrm{C}_{26} \mathrm{H}_{25} \mathrm{~N}_{2} \mathrm{O}_{3} \mathrm{P}: \mathrm{C}$ 70.26, H 5.67, N 6.30; found C 70.15, H $5.77, \mathrm{~N} 6.25$.

\section{2 .3 化合物 13 的合成}

将上步所得 $12(22.40 \mathrm{~g}, 50.4 \mathrm{mmol})$ 加入反应瓶中, 再加入乙醇 $(500 \mathrm{~mL})$ 和水合肼 $(50.4 \mathrm{~mL})$, 随后将反应瓶 放于油浴中进行加热, 回流 $1 \mathrm{~h}$ 后冷却至室温. 将所得 反应液进行减压浓缩得到固体产物. 将所得固体产物依 次加入无水乙醚和二氯甲烷各 $1000 \mathrm{~mL}$ ，常温搅拌 $0.5 \mathrm{~h}$ 后进行过滤. 所得滤液使用无水硫酸美进行搅拌干燥、 抽滤. 滤液经减压浓缩得单磷酰基取代的 $(R, R)$-环已基 二胺 $1315.05 \mathrm{~g}$, 产率 $95 \%$, 无需进一步操作, 密封备 用. m.p. $152 \sim 153{ }^{\circ} \mathrm{C}$; $[\alpha]_{\mathrm{D}}^{21.2}-5.8\left(\right.$ c $\left.2.00, \mathrm{CH}_{2} \mathrm{Cl}_{2}\right)$ (lit. ${ }^{[21]}$ : m.p. $152 \sim 153{ }^{\circ} \mathrm{C} ;[\alpha]_{\mathrm{D}}^{23.4}-6.5\left(c 2.00, \mathrm{CH}_{2} \mathrm{Cl}_{2}\right)$ ); ${ }^{1} \mathrm{H}$ NMR $\left(400 \mathrm{MHz}, \mathrm{CDCl}_{3}\right) \delta: 0.80 \sim 0.95(\mathrm{~m}, 1 \mathrm{H})$, $0.95 \sim 1.22(\mathrm{~m}, 3 \mathrm{H}), 1.44 \sim 1.55(\mathrm{~m}, 2 \mathrm{H}), 1.62 \sim 1.74(\mathrm{br}$, $2 \mathrm{H}), 1.74 \sim 1.85(\mathrm{~m}, 1 \mathrm{H}), 1.87 \sim 2.02(\mathrm{~m}, 1 \mathrm{H}), 2.18 \sim 2.31$ $(\mathrm{m}, 1 \mathrm{H}), 2.36 \sim 2.52(\mathrm{~m}, 1 \mathrm{H}), 3.40 \sim 3.52(\mathrm{~m}, 1 \mathrm{H}), 7.22 \sim$ $7.42(\mathrm{~m}, 6 \mathrm{H}), 7.70 \sim 7.89(\mathrm{~m}, 4 \mathrm{H}) ;{ }^{13} \mathrm{C}$ NMR $(100 \mathrm{MHz}$, $\left.\mathrm{CDCl}_{3}\right) \delta: 24.6,25.0,34.6,34.8(\mathrm{~d}, J=4.5 \mathrm{~Hz}), 56.4(\mathrm{~d}$, $J=4.0 \mathrm{~Hz}), 58.6(\mathrm{~d}, J=2.0 \mathrm{~Hz}), 128.3(\mathrm{~d}, J=12.5 \mathrm{~Hz})$, 131.6 (d, $J=2.4 \mathrm{~Hz}), 131.8,131.9,132.2(\mathrm{~d}, J=9.4 \mathrm{~Hz})$, 132.8 (d, $J=129.9 \mathrm{~Hz}), 133.4(\mathrm{~d}, J=128.0 \mathrm{~Hz}) ;{ }^{31} \mathrm{P}$ NMR $\left(162 \mathrm{MHz}, \mathrm{CDCl}_{3}\right) \delta: 22.2$; IR (NaCl) v: 3162, 3159, 2928, $2854,1435,1180,1123,1108,768 \mathrm{~cm}^{-1}$. HMRS calcd for $\mathrm{C}_{18} \mathrm{H}_{23} \mathrm{~N}_{2} \mathrm{OP}: 314.1548$, found 314.1544. Anal. calcd for $\mathrm{C}_{18} \mathrm{H}_{23} \mathrm{~N}_{2} \mathrm{OP}$ : C 68.77, H 7.37, N 8.91; found C 68.92, H $7.54, \mathrm{~N} 8.64$.

\section{2 .4 化合物 $9 \mathrm{c}$ 的合成}

室温下依次将上述所得 $\mathbf{1 3}(628 \mathrm{mg}, 2.0 \mathrm{mmol}) 、 乙$ 腈 $(10 \mathrm{~mL})$ 、碳酸钾 $(552 \mathrm{mg}, 4.0 \mathrm{mmol}) 、$ 碘代异丙烷(478 $\mathrm{mg}, 2.6 \mathrm{mmol}$ )加入反应瓶中, 加热回流并搅拌过夜. 反 应完成后降温至室温，反应液减压浓缩至无明显馏分得 
到油状物, 向所得油状物中加入二氯甲烷和纯化水各 $20 \mathrm{~mL}$, 搅拌萃取, 分液, 收集有机相, 水相使用二氯甲 烷(30 mL $\times 3)$ 萃取, 合并有机相. 所得有机相使用饱和 食盐水洗涤, 无水硫酸镁干燥后过滤, 滤液浓缩后对所 得物进行柱层析法 $[V$ (甲醇 $) / V$ (二氯甲烷 $)=1 / 40]$ 分离提 纯得配体 9c $563 \mathrm{mg}$, 产率 79\%. m.p. $113 \sim 114{ }^{\circ} \mathrm{C}$; $[\alpha]_{\mathrm{D}}^{22.0}-42.4\left(c 1.00, \mathrm{CH}_{2} \mathrm{Cl}_{2}\right)\left(\right.$ lit. ${ }^{[21]}$ : m.p. $112 \sim 114{ }^{\circ} \mathrm{C}$; $[\alpha]_{D}^{22.0}-42.0\left(\right.$ c 2.00, $\left.\mathrm{CH}_{2} \mathrm{Cl}_{2}\right)$ ); ${ }^{1} \mathrm{H}$ NMR $(400 \mathrm{MHz}$, $\left.\mathrm{CDCl}_{3}\right) \delta: 0.91 \sim 1.00(\mathrm{~m}, 1 \mathrm{H}), 1.04(\mathrm{~d}, J=6.1 \mathrm{~Hz}, 3 \mathrm{H})$, $1.08(\mathrm{~d}, J=6.3 \mathrm{~Hz}, 3 \mathrm{H}), 1.16 \sim 1.30(\mathrm{~m}, 3 \mathrm{H}), 1.54 \sim 1.72$ $(\mathrm{m}, 3 \mathrm{H}), 2.01 \sim 2.11(\mathrm{~m}, 2 \mathrm{H}), 2.27 \sim 2.38(\mathrm{~m}, 1 \mathrm{H}), 2.77 \sim$ $2.88(\mathrm{~m}, 1 \mathrm{H}), 2.88 \sim 3.00(\mathrm{~m}, J=6.2,1 \mathrm{H}), 3.83 \sim 4.08(\mathrm{br}$, $1 \mathrm{H}), 7.39 \sim 7.56(\mathrm{~m}, 6 \mathrm{H}), 7.80 \sim 8.00(\mathrm{~m}, 4 \mathrm{H}) ;{ }^{13} \mathrm{C} \mathrm{NMR}$ $\left(100 \mathrm{MHz}, \mathrm{CDCl}_{3}\right) \delta: 22.8,24.5,24.8,24.9,32.4,34.4$, 45.7, 56.0, 60.0 (d, $J=6.8 \mathrm{~Hz}), 128.3(\mathrm{~d}, J=12.8 \mathrm{~Hz})$, $128.5(\mathrm{~d}, J=12.5 \mathrm{~Hz}), 131.5(\mathrm{~d}, J=2.7 \mathrm{~Hz}), 131.6,131.7$, $132.0(\mathrm{~d}, J=9.5 \mathrm{~Hz}), 133.7$ (d, $J=125.7 \mathrm{~Hz}), 134.0$ (d, $J=130.0 \mathrm{~Hz}) ;{ }^{31} \mathrm{P}$ NMR $\left(162 \mathrm{MHz}, \mathrm{CDCl}_{3}\right) \delta: 22.4$; IR (KBr) $v: 3162,3154,2931,2860,1431,1386,1377,1179$, $1128,1112,764 \mathrm{~cm}^{-1}$. HMRS calcd for $\mathrm{C}_{21} \mathrm{H}_{29} \mathrm{~N}_{2} \mathrm{OP}$ : 356.2018, found 356.2019. Anal. calcd for $\mathrm{C}_{21} \mathrm{H}_{29} \mathrm{~N}_{2} \mathrm{OP}$ : C 70.76, H 8.20, N 7.86; found C 70.80, H 8.03, N 7.86.

\section{3 醛的不对称芳基化反应的基本操作}

将苯嗍酸及其衍生物 $(1.0 \mathrm{mmol})$ 和甲苯加入到干燥 的反应管中, 用氮气置换空气 $5 \sim 6$ 次. 将反应管放于冰 水浴中, 在摚拌条件下将相应的烷基锌试剂( $5.0 \mathrm{~mL}, 1$ $\mathrm{mol} / \mathrm{L}$ 的已烷溶液)缓慢加入到反应管中. 加毕, 将反应 管放于 $60{ }^{\circ} \mathrm{C}$ 的油浴中加热摚拌 $12 \mathrm{~h}$. 反应完全后将所 得芳基烷基锌溶液降至室温备用. 取另一干燥的反应 管, 将手性配体 $(0.15 \mathrm{mmol})$ 和甲苯 $(3 \mathrm{~mL})$ 加入并用氮气 置换空气 5 6 次. 将所得芳基烷基锌试剂加入到反应 管中, 在室温条件下搅拌反应 $30 \mathrm{~min}$, 再将反应管放于 低温反应槽中摚拌 $10 \mathrm{~min}$, 最后将相应的醛 $(0.5 \mathrm{mmol})$ 加入, 搅拌反应 $24 \mathrm{~h}$. 反应结束后, 在 $-40{ }^{\circ} \mathrm{C}$ 条件下逐 滴滴加 $1 \mathrm{~mol} / \mathrm{L}$ 的稀盐酸 $(5 \mathrm{~mL})$ 进行淬灭, 乙酸乙酯 $(10$ $\mathrm{mL} \times 3$ )萃取. 合并有机相, 并用无水硫酸镁干燥, 减压 浓缩, 将粗品进行柱层析提纯 $[V$ ( 乙酸乙酯) $/ V$ (正己 烷 $)=1 / 10$ 得到相应的二芳基甲醇类化合物 $\mathbf{1 0}$. 通过高 效液相色谱法(HPLC)或气相色谱法(GC)对相应产物的 $e e$ 值进行测试, 分子构型通过旋光仪进行旋光度的测试 并与文献报道相对比得出.

\section{4 二芳基甲醇类化合物 10 的表征数据}

$(R)$-对甲苯基苯甲醇 (10a): $[\alpha]_{\mathrm{D}}^{30}+3.8$ (c 1.00, $\left.\mathrm{CHCl}_{3}\right)\left(\right.$ lit. $\left.{ }^{[22]}[\alpha]_{\mathrm{D}}^{25}-5\left(c 1.00, \mathrm{CHCl}_{3}\right)\right) ;{ }^{1} \mathrm{H}$ NMR $(400$ $\left.\mathrm{MHz}, \mathrm{CDCl}_{3}\right) \delta: 2.42(\mathrm{~s}, 3 \mathrm{H}), 3.15(\mathrm{br}, 1 \mathrm{H}), 5.80(\mathrm{~s}, 1 \mathrm{H})$,
$7.18 \sim 7.24(\mathrm{~m}, 2 \mathrm{H}), 7.29 \sim 7.36(\mathrm{~m}, 3 \mathrm{H}), 7.37 \sim 7.46(\mathrm{~m}$, $4 \mathrm{H}) ;{ }^{13} \mathrm{C}$ NMR $\left(100 \mathrm{MHz}, \mathrm{CDCl}_{3}\right) \delta: 21.2,76.1,126.5$, 127.5, 128.5, 129.2, 137.3, 141.0, 144.0; IR (KBr) v: 3270, $2920,1270,1030 \mathrm{~cm}^{-1}$.

$(R)$-对甲氧基苯基苯甲醇 $(\mathbf{1 0 b})$ : $[\alpha]_{\mathrm{D}}^{26.2}+6.6$ (c 1.0, $\left.\mathrm{CHCl}_{3}\right)\left(\right.$ lit. ${ }^{[22]}:[\alpha]_{\mathrm{D}}^{20}-19\left(c 1.0, \mathrm{CHCl}_{3}\right) ;{ }^{1} \mathrm{H}$ NMR $(400$ $\left.\mathrm{MHz}, \mathrm{CDCl}_{3}\right) \delta: 2.32(\mathrm{~d}, J=3.1 \mathrm{~Hz}, 1 \mathrm{H}), 3.81(\mathrm{~s}, 3 \mathrm{H})$, $5.83(\mathrm{~s}, 1 \mathrm{H}), 6.85 \sim 6.96(\mathrm{~m}, 2 \mathrm{H}), 7.23 \sim 7.495(\mathrm{~m}, 7 \mathrm{H})$; ${ }^{13} \mathrm{C}$ NMR $\left(100 \mathrm{MHz}, \mathrm{CDCl}_{3}\right): \delta 55.3,75.8,113.9,126.4$, 127.2, 127.5, 127.9, 128.5, 136.2, 144.0, 159.0; IR (KBr) $v: 3415,3062,2954,2838,1610,1508,1454,1300,1176$, $1031,923 \mathrm{~cm}^{-1}$.

$(R)$ - 对氟苯基苯甲醇 $(10 c)$ : $[\alpha]_{\mathrm{D}}^{27}-6.3$ (c 1.0, $\left.\mathrm{CHCl}_{3}\right)\left(\right.$ lit. ${ }^{[22]}:[\alpha]_{\mathrm{D}}^{25}+8\left(c\right.$ 1.0, $\left.\mathrm{CHCl}_{3}\right) ;{ }^{1} \mathrm{H}$ NMR $(400$ $\left.\mathrm{MHz}, \mathrm{CDCl}_{3}\right) \delta: 2.33(\mathrm{~d}, J=3.6 \mathrm{~Hz}, 1 \mathrm{H}), 5.85(\mathrm{~d}, J=3.0$ $\mathrm{Hz}, 1 \mathrm{H}), 6.90 \sim 7.70(\mathrm{~m}, 9 \mathrm{H}) ;{ }^{13} \mathrm{C}$ NMR $(100 \mathrm{MHz}$, $\left.\mathrm{CDCl}_{3}\right) \delta: 75.6,115.2,115.4,126.5,127.8,128.2,128.3$, 128.6, 139.6, 143.7, 160.9, 163.4; IR (KBr) $v: 3412,1601$, $1505,1219,1167,1025,925,854 \mathrm{~cm}^{-1}$.

$(R)$ - 间澳苯基苯甲醇 $(\mathbf{1 0 d}):[\alpha]_{D}^{28}-10.3$ (c 2 2.0, $\left.\mathrm{CHCl}_{3}\right)\left(\right.$ lit. ${ }^{[23]}:[\alpha]_{D}^{25}+26\left(c 1.0, \mathrm{CHCl}_{3}\right) ;{ }^{1} \mathrm{H}$ NMR $(400$ $\left.\mathrm{MHz}, \mathrm{CDCl}_{3}\right) \delta: 2.56(\mathrm{~s}, 1 \mathrm{H}), 5.79(\mathrm{~s}, 1 \mathrm{H}), 7.18 \sim 7.45(\mathrm{~m}$, $8 \mathrm{H}), 7.53 \sim 7.63(\mathrm{~m}, 1 \mathrm{H}) ;{ }^{13} \mathrm{C}$ NMR $\left(100 \mathrm{MHz}, \mathrm{CDCl}_{3}\right) \delta$ : $75.6,115.3,120.7,122.6,125.1,126.6,128.0,128.8$, 129.6, 130.1, 130.6, 143.2, 146.0; IR (KBr) v: 3383, 1584, $1217,1179,1078,1029,913,758 \mathrm{~cm}^{-1}$.

$(R)$-邻甲基苯基苯甲醇 $(\mathbf{1 0 e})$ : $[\alpha]_{\mathrm{D}}^{25}+6.3$ (c 2.0 , $\left.\mathrm{CHCl}_{3}\right)\left(\right.$ lit. ${ }^{[24]}:[\alpha]_{\mathrm{D}}^{25}+6.8\left(\mathrm{c} 1.0, \mathrm{CHCl}_{3}\right) ;{ }^{1} \mathrm{H}$ NMR $(400$ $\left.\mathrm{MHz}, \mathrm{CDCl}_{3}\right) \delta: 2.19$ (br, $\left.1 \mathrm{H}\right), 2.28(\mathrm{~s}, 3 \mathrm{H}), 6.03(\mathrm{~s}, 1 \mathrm{H})$, $7.12 \sim 7.61(\mathrm{~m}, 9 \mathrm{H}) ;{ }^{13} \mathrm{C}$ NMR $\left(100 \mathrm{MHz}, \mathrm{CDCl}_{3}\right) \delta: 19.4$, $73.4,126.1,126.3,127.1,127.5,127.6,128.5,130.5$, 135.4, 141.5, 142.9; IR (KBr) v: 3365, 3064, 3030, 2919, $1955,1584,1445,1391,1184,1024,951 \mathrm{~cm}^{-1}$.

$(R)$-邻甲氧基苯基苯甲醇 $(\mathbf{1 0 f})$ : $[\alpha]_{\mathrm{D}}^{25.7}+16.3$ (c 1.0, $\left.\mathrm{CHCl}_{3}\right)\left(\right.$ lit. ${ }^{[25]}:[\alpha]_{\mathrm{D}}^{20}+22.8\left(c 1.3, \mathrm{CHCl}_{3}\right) ;{ }^{1} \mathrm{H}$ NMR $(400$ $\left.\mathrm{MHz}, \mathrm{CDCl}_{3}\right) \delta: 3.22(\mathrm{~d}, J=4.2 \mathrm{~Hz}, 1 \mathrm{H}), 3.84(\mathrm{~s}, 3 \mathrm{H})$, $6.12(\mathrm{~s}, 1 \mathrm{H}), 6.90 \sim 7.05(\mathrm{~m}, 2 \mathrm{H}), 7.27 \sim 7.49(\mathrm{~m}, 7 \mathrm{H}) ;{ }^{13} \mathrm{C}$ NMR $\left(100 \mathrm{MHz}, \mathrm{CDCl}_{3}\right) \delta: 55.5,72.2,110.7,121.1$, $123.8,126.6,127.2,127.8,128.2,128.7,132.2,136.1$, 143.5, 149.8, 156.7; IR (KBr) v: 3412, 2905, 2835, 1587, 1491, 1464, 1437, 1242, 1184, 1112, 1026, 856, 754, 729, $698,652 \mathrm{~cm}^{-1}$.

$(R)$ - 间氟苯基苯甲醇 $(\mathbf{1 0 g})$ ： $[\alpha]_{\mathrm{D}}^{26.5}-14.3$ (c 1.0 , $\left.\mathrm{CHCl}_{3}\right)\left(\right.$ lit. ${ }^{[26]}:[\alpha]_{\mathrm{D}}^{22}-10.3\left(c 1.3, \mathrm{CHCl}_{3}\right) ;{ }^{1} \mathrm{H}$ NMR $(400$ $\left.\mathrm{MHz}, \mathrm{CDCl}_{3}\right) \delta: 3.54(\mathrm{~s}, 1 \mathrm{H}), 5.74(\mathrm{~s}, 1 \mathrm{H}), 6.97 \sim 7.10(\mathrm{~m}$, 
$1 \mathrm{H}), 7.12 \sim 7.26(\mathrm{~m}, 2 \mathrm{H}), 7.28 \sim 7.54(\mathrm{~m}, 6 \mathrm{H}) ;{ }^{13} \mathrm{C} \mathrm{NMR}$ $\left(100 \mathrm{MHz}, \mathrm{CDCl}_{3}\right) \delta: 75.7,113.3,113.5,114.2,114.5$, $122.0,126.6,128.0,128.7,129.9,130.0,143.3,146.3$, 161.7, 164.2; IR (KBr) v: 3300, 1600, 1498, $1050 \mathrm{~cm}^{-1}$.

$(R)$-对溴苯基苯甲醇 (10h): $[\alpha]_{\mathrm{D}}^{25}-13.3$ (c 1.0, $\mathrm{CHCl}_{3}$ ) (lit. ${ }^{[26]}:[\alpha]_{\mathrm{D}}^{26.5}-15.2\left(c \quad 1.0, \mathrm{CHCl}_{3}\right){ }^{1} \mathrm{H} \mathrm{NMR}$ $\left(400 \mathrm{MHz}, \mathrm{CDCl}_{3}\right) \delta: 2.20(\mathrm{~d}, J=3.5 \mathrm{~Hz}, 1 \mathrm{H}), 5.81(\mathrm{~d}, J=$ $3.5 \mathrm{~Hz}, 1 \mathrm{H}), 7.25 \sim 7.31(\mathrm{~m}, 3 \mathrm{H}), 7.33 \sim 7.36(\mathrm{~m}, 4 \mathrm{H})$, $7.44 \sim 7.48(\mathrm{~m}, 2 \mathrm{H}) ;{ }^{13} \mathrm{C} \mathrm{NMR}\left(100 \mathrm{MHz}, \mathrm{CDCl}_{3}\right) \delta: 76.0$, $121.7,126.8,128.0,128.2,128.7,131.6,143.1,143.6$; IR (KBr) v: 2138, 1904, 1591, 1483, 1451, 1398, 1237, 1182, 1105, 1070, 1034, 1008, 917, 843, 789, 749, $697 \mathrm{~cm}^{-1}$.

$(R)$ - 对氯苯基苯甲醇 (10i): $[\alpha]_{\mathrm{D}}^{27.4}-5.3$ (c 1.0, $\mathrm{CHCl}_{3}$ ) (lit. ${ }^{[22]}:[\alpha]_{\mathrm{D}}^{25}+19\left(c\right.$ 1.0, $\left.\mathrm{CHCl}_{3}\right){ }^{1} \mathrm{H}$ NMR (400 $\left.\mathrm{MHz}, \mathrm{CDCl}_{3}\right) \delta: 3.68(\mathrm{~s}, 1 \mathrm{H}), 5.67(\mathrm{~s}, 1 \mathrm{H}), 7.26 \sim 7.33(\mathrm{~m}$, 2H), $7.34 \sim 7.49(\mathrm{~m}, 7 \mathrm{H}) ;{ }^{13} \mathrm{C}$ NMR $\left(100 \mathrm{MHz}, \mathrm{CDCl}_{3}\right) \delta$ : 75.6, 126.6, 127.9, 128.6, 128.7, 133.3, 142.4, 143.4; IR (KBr) $v: 3300,1608,1500,1048 \mathrm{~cm}^{-1}$.

$(R)$-对三氟甲基苯基苯甲醇 $(\mathbf{1 0 j})$ : $[\alpha]_{\mathrm{D}}^{25.6}-23.3(c$ 1.0, $\mathrm{CHCl}_{3}$ ) (lit. ${ }^{[22]}:[\alpha]_{\mathrm{D}}^{25}+27\left(c\right.$ 1.0, $\left.\mathrm{CHCl}_{3}\right) ;{ }^{1} \mathrm{H} \mathrm{NMR}$ $\left(400 \mathrm{MHz}, \mathrm{CDCl}_{3}\right) \delta: 3.22(\mathrm{~s}, 1 \mathrm{H}), 5.79(\mathrm{~m}, 1 \mathrm{H}), 7.31 \sim$ $7.43(\mathrm{~m}, 5 \mathrm{H}), 7.45 \sim 7.56(\mathrm{~m}, 2 \mathrm{H}), 7.57 \sim 7.73(\mathrm{~m}, 2 \mathrm{H})$; ${ }^{13} \mathrm{C}$ NMR (100 MHz, $\left.\mathrm{CDCl}_{3}\right) \delta: 75.7,124.2$ (q, $J=272.1$ $\mathrm{Hz}), 125.4$ (q, $J=3.8 \mathrm{~Hz}), 126.69,126.71,128.1,128.8$, 129.6 (q, $J=32.4 \mathrm{~Hz}), 143.1,147.5$; IR $(\mathrm{KBr}) v$ : 3252, 1620, 1601, 1494, 1450, 1418, 1335, 1111, 1069, 1016, $924,866,849,818,731,702,534,457 \mathrm{~cm}^{-1}$.

$(R)$-1- 䒺 基 苯甲醇 $(\mathbf{1 0 k}):[\alpha]_{\mathrm{D}}^{24.6}+25.6$ (c 1.0, $\left.\mathrm{CHCl}_{3}\right)\left(\right.$ lit $^{[22]}:[\alpha]_{\mathrm{D}}^{25}-42\left(c 1.0, \mathrm{CHCl}_{3}\right) ;{ }^{1} \mathrm{H}$ NMR $(400$ $\left.\mathrm{MHz}, \mathrm{CDCl}_{3}\right) \delta: 3.00(\mathrm{~s}, 1 \mathrm{H}), 6.48(\mathrm{~s}, 1 \mathrm{H}), 7.27 \sim 7.61(\mathrm{~m}$, $8 \mathrm{H}), 7.63 \sim 7.72(\mathrm{~m}, 1 \mathrm{H}), 7.83 \sim 8.00(\mathrm{~m}, 2 \mathrm{H}), 8.02 \sim 8.13$ $(\mathrm{m}, 1 \mathrm{H}) ;{ }^{13} \mathrm{C}$ NMR $\left(100 \mathrm{MHz}, \mathrm{CDCl}_{3}\right) \delta: 73.6,124.1$, $124.7,125.4,125.7,126.2,127.2$, 127.7, 128.5, 128.6, $128.9,129.6,130.8,134.0,138.9,143.2$, IR (KBr) v: 3345, 3053, 2927, 1603, 1514, 1490, 1457, 1182, 1052, 993, 794, $771,710 \mathrm{~cm}^{-1}$.

(R)-2-菜基苯甲醇(101): $[\alpha]_{\mathrm{D}}^{28.4}-5.5\left(c \quad 1.0, \mathrm{CHCl}_{3}\right)$ (lit. ${ }^{[22]}:[\alpha]_{\mathrm{D}}^{25}+6\left(c\right.$ 1.0, $\left.\mathrm{CHCl}_{3}\right){ }^{1} \mathrm{H} \mathrm{NMR}(400 \mathrm{MHz}$, $\left.\mathrm{CDCl}_{3}\right) \delta: 3.00(\mathrm{~s}, 1 \mathrm{H}), 6.00(\mathrm{~s}, 1 \mathrm{H}), 7.28 \sim 7.64(\mathrm{~m}, 8 \mathrm{H})$, $7.77 \sim 8.00(\mathrm{~m}, 4 \mathrm{H}) ;{ }^{13} \mathrm{C} \mathrm{NMR}\left(100 \mathrm{MHz}, \mathrm{CDCl}_{3}\right) \delta: 76.3$, $124.9,125.2,126.1,126.2,126.8,127.7,127.8,128.2$, 128.4, 128.6, 133.0, 133.3, 143.7; IR (KBr) v: 3384, 3057, 2874, 1951, 1597, 1497, 1366, 1160, 1028, $957 \mathrm{~cm}^{-1}$.

$(R)$ - 对氯苯基苯甲醇 $(\mathbf{1 0 m})$ : $[\alpha]_{\mathrm{D}}^{24.3}-9.4$ (c 1.0, $\left.\mathrm{CHCl}_{3}\right)$ (lit. ${ }^{[26]}:[\alpha]_{\mathrm{D}}^{22.2}-8.8\left(c 1.0, \mathrm{CHCl}_{3}\right) ;{ }^{1} \mathrm{H}$ NMR (400
$\left.\mathrm{MHz}, \mathrm{CDCl}_{3}\right) \delta:{ }^{13} \mathrm{C}$ NMR $\left(100 \mathrm{MHz}, \mathrm{CDCl}_{3}\right) \delta: 148.8$, $140.3,135.2,133.7,131.8,128.0,127.6,127.5,127.3$, $127.0,126.8(\mathrm{~d}, J=6.4 \mathrm{~Hz}), 126.0,125.5,125.5,125.1$, 75.3, 34.2, 31.3 (t, $J=5.5 \mathrm{~Hz}$ ); IR (KBr) v: 3342, 3044, $2870,1947,1395,1365,963 \mathrm{~cm}^{-1}$.

$(R)$-2-菜基对叔丁基苯甲醇(10n): $[\alpha]_{\mathrm{D}}^{24.5}-10.7$ (c 1.0, $\left.\mathrm{CHCl}_{3}\right)\left(\right.$ lit. ${ }^{[26]}$ : $[\alpha]_{\mathrm{D}}^{22.9}-8.2\left(c 1.0, \mathrm{CHCl}_{3}\right) ;{ }^{1} \mathrm{H} \mathrm{NMR}$ $\left(400 \mathrm{MHz}, \mathrm{CDCl}_{3}\right) \delta: 7.51(\mathrm{~s}, 1 \mathrm{H}), 7.39 \sim 7.37(\mathrm{~m}, 1 \mathrm{H})$, $7.30 \sim 7.23(\mathrm{~m}, 3 \mathrm{H}), 7.18(\mathrm{t}, J=7.6 \mathrm{~Hz}, 1 \mathrm{H}), 7.00(\mathrm{t}, J=$ $8.4 \mathrm{~Hz}, 2 \mathrm{H}), 5.74(\mathrm{~s}, 1 \mathrm{H}), 2.18(\mathrm{~s}, 1 \mathrm{H}) .{ }^{13} \mathrm{C}$ NMR $(100$ $\left.\mathrm{MHz}, \mathrm{CDCl}_{3}\right) \delta: 160.4,142.4,139.0,133.0,130.4,130.0$, $129.8,129.1,127.2,123.6,116.0,115.7,74.2$; IR (KBr) $v$ : $3412,3383,1601,1505,1219,1167,1025,925,854,1217$ $\mathrm{cm}^{-1}$.

$(S)$-对甲酸甲酯基对氟苯甲醇 $(\mathbf{1 0 0})$ : $[\alpha]_{\mathrm{D}}^{23.6}-11.9$ (c 1.0, $\mathrm{CHCl}_{3}$ ) (lit. ${ }^{[26]}: \quad[\alpha]_{\mathrm{D}}^{22.5}-11.4\left(\right.$ c $\left.1.0, \mathrm{CHCl}_{3}\right) ;{ }^{1} \mathrm{H}$ NMR (400 MHz, $\left.\mathrm{CDCl}_{3}\right) \delta: 7.97(\mathrm{~d}, J=8.4 \mathrm{~Hz}, 2 \mathrm{H}), 7.45$ $(\mathrm{d}, J=8.0 \mathrm{~Hz}, 2 \mathrm{H}), 7.33 \sim 7.25(\mathrm{~m}, 5 \mathrm{H}), 5.85(\mathrm{~s}, 1 \mathrm{H}), 3.87$ $(\mathrm{s}, 3 \mathrm{H}), 2.40(\mathrm{~s}, 1 \mathrm{H}) ;{ }^{13} \mathrm{C} \mathrm{NMR}\left(100 \mathrm{MHz}, \mathrm{CDCl}_{3}\right) \delta$ : $166.9,148.8,143.1,129.5,128.7,128.4,127.6,126.5$, 126.2, 75.5, 51.9; IR (KBr) v: 3467, 3023, 3012, 2911, 1943, 1712, 1576, 1354, 1321, 1074, $1021,931 \mathrm{~cm}^{-1}$.

$(S)$-对甲基对氟苯甲醇(10p): $[\alpha]_{\mathrm{D}}^{23.2}+6.5$ (c 1.0, $\mathrm{CHCl}_{3}$ ) (lit. ${ }^{[26]}:[\alpha]_{\mathrm{D}}^{24}+6.2\left(c 1.0, \mathrm{CHCl}_{3}\right) ;{ }^{1} \mathrm{H}$ NMR $(400$ $\left.\mathrm{MHz}, \mathrm{CDCl}_{3}\right) \delta: 7.36 \sim 7.30(\mathrm{~m}, 2 \mathrm{H}), 7.24(\mathrm{~d}, J=8.3 \mathrm{~Hz}$, 2H), $7.17(\mathrm{~d}, J=7.9 \mathrm{~Hz}, 2 \mathrm{H}), 7.06 \sim 6.98(\mathrm{~m}, 2 \mathrm{H}), 5.76(\mathrm{~s}$, 1H), 2.49 (br 1H), $2.36(\mathrm{~s}, 3 \mathrm{H}) ;{ }^{13} \mathrm{C} \mathrm{NMR}(100 \mathrm{MHz}$, $\left.\mathrm{CDCl}_{3}\right) \delta: 140.8,139.7,137.4,129.2,128.1,126.4,115.3$, 115.0, 75.3, 21.0; IR (KBr) v: 3178, 2960, 2870, 1609, $1501,1460,1382,763 \mathrm{~cm}^{-1}$.

$(R)$-均三甲苯基对甲基苯甲醇 $(\mathbf{1 0 q})$ : $[\alpha]_{\mathrm{D}}^{24.7}+42.3$ (c 1.0, $\left.\mathrm{CHCl}_{3}\right)\left(\right.$ lit. $^{[26]}:[\alpha]_{\mathrm{D}}^{24}+39\left(c 1.0, \mathrm{CHCl}_{3}\right) ;{ }^{1} \mathrm{H} \mathrm{NMR}$ $\left(400 \mathrm{MHz}, \mathrm{CDCl}_{3}\right) \delta: 7.29 \sim 7.11(\mathrm{~m}, 3 \mathrm{H}), 7.07 \sim 6.97(\mathrm{~m}$, $2 \mathrm{H}), 6.91(\mathrm{~s}, 2 \mathrm{H}), 4.03(\mathrm{~s}, 2 \mathrm{H}), 2.31(\mathrm{~s}, 3 \mathrm{H}), 2.22(\mathrm{~s}, 6 \mathrm{H})$, $2.15(\mathrm{~s}, 3 \mathrm{H}) ;{ }^{13} \mathrm{C} \mathrm{NMR}\left(100 \mathrm{MHz}, \mathrm{CDCl}_{3}\right) \delta: 141.5,135.4$, $135.1,140.3,136.2,135.7,130.3,129.4,128.1,128.6$, 127.1 126.8, 71.2, 21.9, 21.3, 20.0, 19.8; IR (KBr) v: 3403, 2896, 1611, 1584, 1493, 1441, 1373, 1142, 1034, 1007, $892,795,714,687 \mathrm{~cm}^{-1}$.

$(R)$-邻甲基苯基对氯苯甲醇(10r): $[\alpha]_{\mathrm{D}}^{24.5}-6.3(c$ 1.0, $\left.\mathrm{CHCl}_{3}\right)\left(\right.$ lit. $^{[26]}$ : $[\alpha]_{\mathrm{D}}^{23.8}-5.6\left(c 1.0, \mathrm{CHCl}_{3}\right) ;{ }^{1} \mathrm{H} \mathrm{NMR}$ $\left(400 \mathrm{MHz}, \mathrm{CDCl}_{3}\right) \delta: 2.25(\mathrm{~d}, J=3.5 \mathrm{~Hz}, 1 \mathrm{H}), 2.27$ (s, $3 \mathrm{H}), 5.98(\mathrm{~s}, 1 \mathrm{H}), 7.14 \sim 7.35(\mathrm{~m}, 7 \mathrm{H}), 7.43 \sim 7.50(\mathrm{~m}$, $1 \mathrm{H}) ;{ }^{13} \mathrm{C}$ NMR $\left(100 \mathrm{MHz}, \mathrm{CDCl}_{3}\right) \delta: 141.8,141.5,135.8$, $133.7,131.1,129.0,128.8,128.2,126.8,126.7,73.2$, 19.8; 
IR (KBr) v: 3352, 2925, 1488, 1461, 1090, 1013, 752 $\mathrm{cm}^{-1}$.

(R)-2-噻吩基苯甲醇 $(\mathbf{1 0 s}):[\alpha]_{\mathrm{D}}^{22.3}-7.6$ (c 0.59 , $\left.\mathrm{CHCl}_{3}\right)$ (lit. ${ }^{[10]}:[\alpha]_{\mathrm{D}}^{25}+10.0\left(c\right.$ 0.32., $\left.\mathrm{CHCl}_{3}\right) ;{ }^{1} \mathrm{H} \mathrm{NMR}$ $\left(400 \mathrm{MHz}, \mathrm{CDCl}_{3}\right) \delta: 7.42(\mathrm{~d}, J=7.1 \mathrm{~Hz}, 2 \mathrm{H}), 7.35(\mathrm{t}, J=$ $7.3 \mathrm{~Hz}, 2 \mathrm{H}), 7.27 \sim 7.31(\mathrm{~m}, 1 \mathrm{H}), 7.23(\mathrm{dd}, J=5.1,1.1 \mathrm{~Hz}$, $1 \mathrm{H}), 6.92(\mathrm{dd}, J=5.0,3.5 \mathrm{~Hz}, 1 \mathrm{H}), 6.85$ (d, $J=3.5 \mathrm{~Hz}$, $1 \mathrm{H}), 6.00(\mathrm{~s}, 1 \mathrm{H}), 2.60(\mathrm{~s}, 1 \mathrm{H}) ;{ }^{13} \mathrm{C} \mathrm{NMR}(100 \mathrm{MHz}$, $\left.\mathrm{CDCl}_{3}\right) \delta: 148.1,143.1,128.5,128.0,126.7,126.3,125.5$, 124.9, 72.4; IR (KBr) v: 3376, 1594, 1442, 1216, 1038, $759,703,703 \mathrm{~cm}^{-1}$.

辅助材料(Supporting Information) 配体及产物的核 磁共振谱图、GC 和 HPLC 谱图. 这些材料可以免费从 本刊网站(http://sioc-journal.cn/)上下载.

\section{References}

[1] Einhorn, A. Ber. Dtsch. Chem. Ges. 1883, 16, 2208.

[2] Torrens, A.; Castrillo, J.; Claparols, A.; Redondo, J. Synlett 1999, 765.

[3] (a) Casy, A. F.; Drake, A. F.; Ganellin, C. R.; Mercer, A. D.; Upton, C. Chirality 1992, 4, 356. (b) Müller, P.; Nury, P.; Bernardinelli, G. Eur. J. Org. Chem. 2001, 2001, 4137.

[4] Hite, G.; Barouh, V.; Dall, H.; Patel, D. J. Med. Chem. 1971, 14, 834.

[5] Guo, Z.; Raeissi, S.; White, R. B.; Stevens, J. C. Drug Metab. Dispos. 1997, 25, 390.

[6] Li, C.; Chauret, N.; Trimble, L. A.; Nicoll-Griffith, D. A; Silva, J. M; MacDonald, D.; Perrier, H.; Yergey, J. A.; Parton, T.; Alexander, R. P.; Warrellow, G. J. Drug Metab. Dispos. 2001, 29, 232.

[7] (a) James, M. N. G.; Williams, G. J. B. Can. J. Chem. 1974, 52, 1872 .

(b) Shafi'ee, A.; Hite, G. J. Med. Chem. 1969, 12, 266.

[8] (a) Bolm, C.; Hildebrand, J. P.; Muñiz, K.; Hermanns, N. Angew. Chem., Int. Ed. 2001, 40, 3284. (b) Ji, J. X.; Wu, J.; Xu, L. J.; Chiu, W. Y.; Kim, H. L.; Albert, S. C. Pure Appl. Chem. 2006; 78, 267.

[9] (a) Fernández-Mateos, E.; Maciá, B.; Yus, M. Tetrahedron: Asymmetry 2012, 23, 789.

(b) Wu, K. H.; Gau, H. M. J. Am. Chem. Soc. 2006, 128, 14808.

[10] (a) Da, C.-S.; Wang, J. R.; Yin, X. G.; Fan, X. Y.; Liu, Y.; Yu, S. L. Org. Lett. 2009, 11, 5578.

(b) Fan, X. Y.; Yang, Y. X.; Zhuo, F.-F.; Yu, S. L.; Li, X.; Guo, Q. P.; Du, Z. X.; Da, C. S. Chem.-Eur. J. 2010, 16, 7988.

[11] Tomita, D.; Wada, R.; Kanai, M.; Shibasaki, M. J. Am. Chem. Soc. 2005, 127, 4138 .

[12] Chang, S. J.; Zhou, S.; Gau, H. M. RSC Adv. 2015, 5, 9368.

[13] (a) Fernández-Mateos, E.; Maciá, B.; Yus, M. Eur. J. Org. Chem. 2012, 3732 .

(b) Nakagawa, Y.; Muramatsu, Y.; Harada, T. Eur. J. Org. Chem. 2010, 6535 .

[14] Bolm, C.; Rudolph, J. J. Am. Chem. Soc. 2002, 124, 14850.

[15] Bauer, M.; Maurer, F.; Hoffmann, S. M.; Kazmaier U. Synlett 2008, 3203.

[16] (a) Ding, M.; Zhou, F.; Liu, Y. L.; Wang, C. H.; Zhao, X. L.; Zhou, J. Chem. Sci. 2011, 2, 2035.

(b) Dong, J.; Du, D. M. Org. Biomol. Chem. 2012, 10, 8125.

(c) Yu, Y. N.; Xu, M. H. Acta Chim. Sinica 2017, 75, 655 (in Chinese).

(于月娜, 徐明华, 化学学报, 2017, 75, 655.)

[17] Huang, H.; Bian, G.; Zong, H.; Wang, Y.; Yang, S.; Yue, H.; Song, L.; Fan, H. Org. Lett. 2016, 18, 2524.

[18] Shen, B.; Huang, H.; Bian, G.; Zong, H.; Song, L. Chirality 2013, 25,561 .

[19] Huang, H. Y.; Zong, H.; Shen, B.; Yue, H. F.; Bian, G. L.; Song, L. Tetrahedron 2014, 70, 1289.

[20] Kaik, M.; Gawroński, J. Tetrahedron: Asymmetry 2003141559.

[21] Huang, H. Y.; Z ong, H.; Bian, G. L.; Song, L. J. Org. Chem. 2012, 77, 10427.

[22] Yang, Y. X.; Liu, Y.; Zhang, L.;Jia, Y. E.; Wang, P.; Zhuo, F. F.; An, X. T.; Da, C. S. J. Org. Chem. 2014, 79, 10696.

[23] Wu, X. Y.; Liu, X. Y.; Zhao, G. Tetrahedron: Asymmetry 2005, 16, 2299.

[24] Tian, C.; Gong, L.; Meggers, E. Chem. Commun. 2016, 52, 4207.

[25] Barsamian, A. L.; Wu, Z. H.; Blakemore, P. R. Org. Biomol. Chem. 2015, 13, 3781.

[26] Wang, Y. B.; Zong, H.; Huang, H. Y.; Song, L. Tetrahedron: Asymmetry 2017, 28, 90. 